(6)

OPEN ACCESS

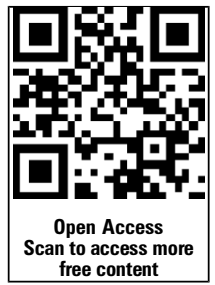

- Additional material is published online only. To view please visit the journal online (http://dx.doi.org/10.1136/ gutjnl-2014-308900)

${ }^{1}$ Department of Hematology, Hokkaido University Graduate School of Medicine, Sapporo, Hokkaido, Japan

${ }^{2}$ Division of Cellular Therapy, Advanced Clinical Research Center, The Institute of Medical Science, The University of Tokyo, Tokyo, Japan ${ }^{3}$ Atopy Research Center, Juntendo University School of Medicine, Tokyo, Japan ${ }^{4}$ Division of Stem Cell Signaling, The Institute of Medical Science, The University of Tokyo, Tokyo, Japan

\section{Correspondence to} Dr Toshio Kitamura, Division of Cellular Therapy, Advanced Clinical Research Center, The Institute of Medical Science, The University of Tokyo, 4-6-1 Shirokanedai, Minato-ku, Tokyo 108-8639, Japan; kitamura@ims.u-tokyo.ac.jp and

Dr Jiro Kitaura, Atopy Research Center, Juntendo University School of Medicine, 2-1-1 Hongo, Bunkyo-ku, Tokyo 113-8421, Japan kitaura-tky@umin.ac.jp

Received 26 November 2014 Revised 11 January 2015 Accepted 17 January 2015 Published Online First 11 February 2015

\title{
Ceramide-CD300f binding suppresses experimental colitis by inhibiting ATP-mediated mast cell activation
}

\author{
Toshihiro Matsukawa, ${ }^{1,2}$ Kumi Izawa, $^{2,3}$ Masamichi Isobe, ${ }^{2}$ Mariko Takahashi, $^{2}$ \\ Akie Maehara, ${ }^{2}$ Yoshinori Yamanishi, ${ }^{2}$ Ayako Kaitani, ${ }^{2}$ Ko Okumura, ${ }^{3}$ \\ Takanori Teshima, ${ }^{1}$ Toshio Kitamura, ${ }^{2,4}$ Jiro Kitaura ${ }^{2,3}$
}

\section{ABSTRACT}

Objective Extracellular ATP mediates mast celldependent intestinal inflammation via P2X7 purinoceptors. We have previously shown that CD300f (also called the leucocyte mono-immunoglobulin-like receptor 3 (LMIR3)) suppresses immunoglobulin E-dependent and mast cell-dependent allergic responses by binding to ceramide. The aim of the present study was to clarify the role of ceramide-LMIR3 interaction in the development of IBD.

Design The dextran sodium sulfate (DSS)-induced colitis model was used in wild-type (WT), LMIR3 ${ }^{-/-}$

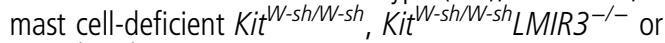
$K i t^{W-s h / W-s h}$ mice engrafted with WT or $L M I R 3^{-/-}$bone marrow-derived mast cells (BMMCs). The severity of colitis was determined by clinical and histological criteria. Lamina propria cell populations were assessed by flow cytometry. Production of chemical mediators from lamina propria cells was measured by real-time reverse transcription PCR. Production of chemical mediators from ATP-stimulated BMMCs in the presence or absence of ceramide was measured by ELISA. The severity of DSS-induced colitis was assessed in mice given either an Fc fusion protein containing an extracellular domain of LMIR3, and anticeramide antibody, or ceramide liposomes.

Results LMIR3 deficiency exacerbated DSS-induced colitis in mice. Kit ${ }^{W-s h / W-s h}$ mice harbouring $L M I R 3^{-/-}$ mast cells exhibited more severe colitis than those harbouring WT mast cells. Ceramide-LMIR3 interaction inhibited ATP-stimulated activation of BMMCs. DSS-induced colitis was aggravated by disrupting the ceramide-LMIR3 interaction, whereas it was suppressed by treating with ceramide liposomes.

Conclusions LMIR3-deficient colonic mast cells were pivotal in the exacerbation of DSS-induced colitis in $\mathrm{LMIR3}^{-/-}$mice. Ceramide liposomes attenuated DSSinduced colitis by inhibiting ATP-mediated activation of colonic mast cells through ceraimide-LMIR3 binding.

\section{INTRODUCTION}

IBD is characterised by dysregulated intestinal inflammation. The incidence and prevalence of IBD, including UC and Crohn's disease, have increased worldwide. IBD is a complex multifactorial disease regulated by the interplay between immunity, environmental factors and genetic susceptibility. ${ }^{1-4}$

\section{Significance of this study}

What is already known on this subject?

- Mast cells are involved in the pathogenesis of IBD.

- Extracellular ATP mediates mast cell-dependent intestinal inflammation via $\mathrm{P} 2 \mathrm{X7}$ purinoceptors.

- An inhibitory receptor LMIR3/CD300f suppresses immunoglobulin E-dependent and mast cell-dependent allergic responses in mice.

What are the new findings?

- LMIR3 deficiency exacerbated dextran sodium sulfate (DSS)-induced colitis in mice.

- Mast cell-deficient $K i t^{W-s h / W-s h}$ mice transplanted with $L M I R 3^{-1-}$ mast cells exhibited more severe colitis than those with wild-type mast cells.

- Ceramide-LMIR3 interaction inhibited ATP-stimulated activation of bone marrow-derived mast cells.

- DSS-induced colitis was aggravated by disrupting the ceramide-LMIR3 interaction, whereas it was suppressed by treating with ceramide liposomes.

How might it impact on clinical practice in the foreseeable future?

- The present study provided evidence that the ceramide-LMIR3 interaction inhibits ATP-mediated activation of colonic mast cells, thereby suppressing the development of experimental colitis. LMIR3-targeted ceramide liposomes would provide novel therapeutic strategies for IBD.

To define the underlying mechanisms, a number of chemically induced mouse models of IBD have been developed. Among them, the dextran sodium sulfate (DSS) or 2,4,6-trinitrobenzene sulfonic acid (TNBS)-induced colitis models have similarities to human UC or Crohn's disease, respectively. ${ }^{5-7}$ Extensive research has revealed that together with intestinal epithelial cells, a variety of colonic innate immune cells, including mast cells, neutrophils, eosinophils and $\mathrm{CD} 11 \mathrm{~b}^{+} \mathrm{CX} 3 \mathrm{CR} 1^{\text {int }}$ mononuclear cells, release an array of chemical mediators (eg, cytokines, 
chemokines, proteases and lipid mediators) in inflammatory cites of the colon. ${ }^{2-4} 8-12$ Dysregulated inflammatory mediators exacerbate acute colitis, although several cytokines promote tissue repair to maintain intestinal homeostasis. ${ }^{2-4}$ Studies with mast celldeficient mice and with P2X7-deficient mast cells have recently demonstrated that ATP-mediated mast cell activation plays a critical role in the initiation and development of experimental colitis induced by DSS and by TNBS; extracellular ATP produced in injured colons activates colonic mast cells via the P2X7 purinoceptor, which release chemical mediators, including neutrophil chemoattractants. ${ }^{8}$ Accordingly, we aimed to identify a negative regulator of ATP-stimulated mast cell activation that would lead to a new therapeutic target for IBD. One of the possible candidates is an inhibitory receptor expressed in mast cells, ${ }^{13-16}$ which suppresses mast cell activation through binding to its specific ligand.

A variety of paired activating and inhibitory receptor families regulate the immune system. ${ }^{14-18}$ The inhibitory receptor CD300f (also called leucocyte mono-immunoglobulin-like receptor 3 (LMIR3), CMRF35-like molecule-1 or myeloidassociated immunoglobulin-like receptor-V) harbours two immunoreceptor tyrosine-based inhibitory motifs (ITIMs) and a single immunoreceptor tyrosine-based switch motif (ITSM). ${ }^{14} 1718$ LMIR3 is expressed in myeloid cells, including mast cells. We have recently identified ceramide as a ligand for LMIR3. ${ }^{15}$ Ceramide-LMIR3 interaction inhibits immunoglobulin E (IgE)-dependent and mast cell-dependent allergic responses via the two ITIMs and single ITSM $;{ }^{14}$ however, its role in other settings of inflammation, such as colitis, remains elusive.

In this study, we demonstrate that $L M I R 3^{-/-}$mice are highly susceptible to experimental colitis. Analyses of mast celldeficient mice adoptively transferred with wild-type (WT) or $\mathrm{LMIR}^{-/-}$bone marrow-derived mast cells (BMMCs) underscore the importance of mast cells in the exacerbation of DSS-induced colitis in $L M I R 3^{-/-}$mice. In addition, our results provide evidence that the ceramide-LMIR3 interaction inhibits ATP-mediated mast cell activation, thereby suppressing the development of experimental colitis.

\section{MATERIALS AND METHODS}

Mice

Female, $\mathrm{LMIR}^{-/-}$mice aged 10 to 12 weeks on the C57BL/6 background (12 generations backcrossed) or on the $\mathrm{BALB} / \mathrm{c}$ background (eight generations backcrossed), C57BL/6 or BALB/ c mice (Charles River Laboratories Japan), Kit ${ }^{W-s h / W-s h}$ mice and $\mathrm{Kit}^{\mathrm{W}-\mathrm{s} h / \mathrm{W}-\mathrm{sh}} \mathrm{LMIR}^{-/-}$mice were used as described. ${ }^{14}{ }^{19}$ All mice were housed under specific pathogen-free conditions. All the procedures were approved by an institutional review committee of the University of Tokyo (approval no 20-8).

\section{Cells}

BMMCs and BMMC transfectants were generated as described. ${ }^{14} 20$ Lamina propria mononuclear cells (LPMNCs) were isolated from the colon as described. ${ }^{8}{ }^{21}$ Briefly, the colon was digested with $1 \mathrm{mg} / \mathrm{mL}$ collagenase type IV (Sigma-Aldrich, St Louis, Missouri, USA) in RPMI-1640 medium. After filtering, the cells were washed, resuspended in the $40 \%$ Percoll (GE Healthcare, Munich, Germany) and overlaid on top of the $75 \%$ Percoll. After centrifugation, the cells at the interface were collected as LPMNCs.

\section{Induction of experimental colitis}

Sibling or non-sibling mice were cohoused for 1 month before induction of experimental colitis. For DSS-induced colitis, mice were given DSS (molecular weight $36-50 \mathrm{kDa}$; MP Biologicals,
Solon, Ohio, USA) supplemented in sterile, distilled water for 7 days followed by normal drinking water until the end of the experiment, as previously described. ${ }^{5} 6{ }^{8}$ For mice on the C57BL/6 background, $1.5 \%$ or $2.5 \%$ (wt $/ \mathrm{vol}$ ) DSS was used to induce colitis. In the case of mice on the BALB/c background, $3 \%(\mathrm{wt} / \mathrm{vol})$ DSS was used to induce colitis. For treatment with antibody $(\mathrm{Ab})$ or Fc fusion protein, mice on the C57BL/6 background were intraperitoneally injected with $5 \mu \mathrm{g}$ of anticeramide $\mathrm{Ab}$ or control $\mathrm{Ab}$ on day 2 after 1.5\% DSS treatment or with $300 \mu \mathrm{g}$ of an extracellular domain of LMIR3 fused to an Fc domain of human IgG1 (LMIR3-Fc) or of control Fc on days 2 and 6 after $1.5 \%$ DSS treatment. For treatment with liposomes, mice on the C57BL/6 background were intravenously injected with $100 \mu \mathrm{g}$ of ceramide or phosphatidylcholine (PC) liposomes on days 2 and 6 after $2.5 \%$ DSS treatment. For TNBS-induced colitis, anaesthetised mice on the C57BL/6 background were sensitised with 2.5\% TNBS (Sigma-Aldrich) together with acetone and olive oil. One week after sensitisation, the mice were intrarectally administered with $100 \mu \mathrm{g}$ of $1.5 \%$ TNBS in $50 \%$ ethanol, as described. ${ }^{5} 78$ A Disease Activity Index (DAI) score was determined by daily assessment of weight loss, stool consistency and bleeding. ${ }^{5-8}$ For histopathological analysis, the entire colon was fixed in $10 \%$ formalin after measuring the colon length on day 7 after DSS treatment. Paraffin-embedded sections were stained with H\&E.

\section{Statistical analyses}

Results are expressed as mean \pm SEM. An unpaired Student's t test was used to compare the differences between groups. The Kaplan-Meier method and log-rank tests were used to analyse survival studies. ${ }^{*} \mathrm{p}<0.05, * \mathrm{p}<0.01$ or $* * \mathrm{p}<0.001$ was taken as statistically significant.

\section{RESULTS}

$\mathrm{LMIR3}^{-/-}$mice are highly susceptible to DSS-induced colitis

To explore the role of the inhibitory receptor LMIR3 in the development of colitis, we used the DSS-induced colitis model in WT and LMIR $3^{-/-}$mice on the C57BL/6 background. The mice were given $1.5 \%$ DSS in the drinking water for 7 days. The clinical signs of colitis, including body weight loss, diarrhoea and rectal bleeding, were monitored daily. On day 7 after DSS treatment, colon length was measured before histological examination. WT mice developed only mild colitis in this model (figure 1A-D). Conversely, LMIR $3^{-/-}$mice exhibited greater weight loss and colon shortening as well as higher DAI scores as compared with WT mice (figure 1A-C). No histological difference was observed in the colonic sections between WT and $L M I R 3^{-/-}$mice prior to DSS treatment, whereas we found a massive destruction of colonic epithelial structures with robust inflammatory cell infiltrates in the mucosa in DSS-treated $L M I R 3^{-/-}$mice compared with WT mice (figure 1D). Notably, 2.5\% DSS treatment induced more severe colitis in WT mice than 1.5\% DSS treatment did (figure 1E; see online supplementary figure S1). WT mice were still protected against DSS-induced lethal colitis during the observation period (figure $1 \mathrm{~F}$ ). In contrast, $>70 \%$ of $L_{M I R 3^{-/-}}$mice died of aggressive colitis with prominent histological alterations and clinical features, including rapid weight loss, within 11 days after $2.5 \%$ DSS treatment (figure 1E, F; see online supplementary figure S1). Similarly, LMIR $3^{-/-}$mice on the $\mathrm{BALB} / \mathrm{c}$ background were more susceptible to DSS-induced colitis than their WT counterparts (see online supplementary figure S2). Similar to DSS treatment, TNBS treatment induced more severe colitis with greater weight loss and colon shortening in $L M I R 3^{-/-}$mice than in WT mice (see online supplementary 



C
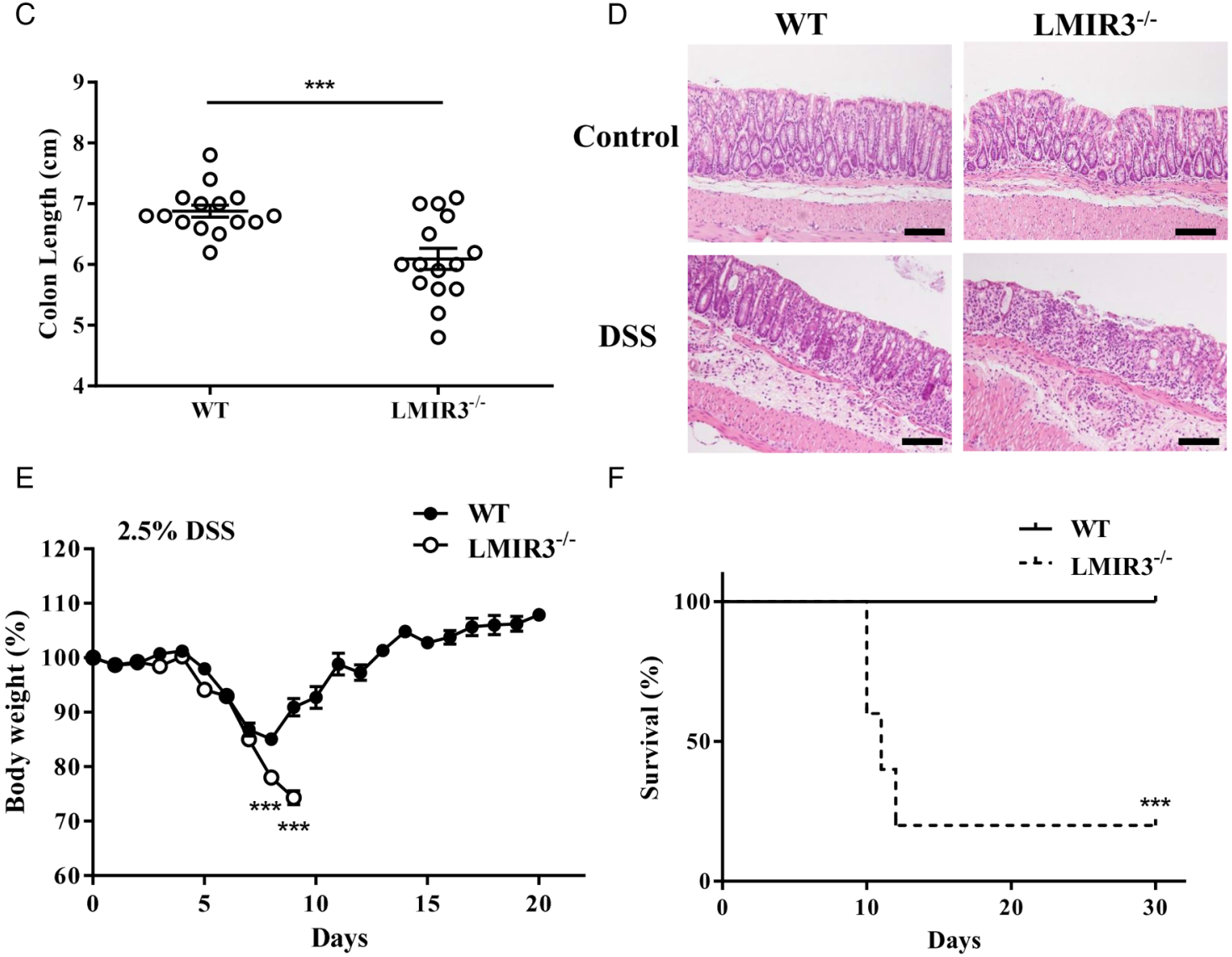

Figure 1 Leucocyte mono-immunoglobulin-like receptor $3(L M I R 3)^{-/-}$mice are highly susceptible to dextran sodium sulfate (DSS)-induced colitis. (A)-(F) Wild-type (WT) or LMIR3 ${ }^{-1-}$ mice on the C57BL/6 background were subjected to (A-D) the 1.5\% DSS-induced colitis model (each, $\mathrm{n}=15$ ) or to $(E$ and $F$ ) the $2.5 \%$ DSS-induced colitis model (each, $n=10$ ). (A and $E$ ) Body weight and (B) Disease Activity Index (DAI) score. (C) Colon length on day 7. (D) Representative H\&E-stained colonic sections are shown. Scale bars represent $100 \mu \mathrm{m}$. (F) Mice were monitored for survival. (A-C and E) Data are expressed as the mean \pm SEM. ${ }^{* *} p<0.01,{ }^{* * *} p<0.001$ (Student's $t$ test). (A-F) The data are from one experiment which is representative of the other one experiment performed $(C)$ or of the other two experiments performed $(A, B, D-F)$.

figure S3). Thus, LMIR3 deficiency exacerbated acute experimental colitis in mice.

DSS-treated $L M I R 3^{-/-}$mice exhibit a robust increase in degranulated mast cells in the colon

For further analysis, we induced colitis using $1.5 \%$ DSS to prevent DSS-induced death in LMIR3 ${ }^{-/}$mice. We then examined LMIR3 expression in myeloid cells within the colonic lamina propria of mice before and after DSS exposure. Surface expression of LMIR3 was detected in the high-affinity $\operatorname{IgE}$ receptor $(\mathrm{FccRI})^{+} \mathrm{c}-\mathrm{kit}^{+}$mast cells, CD11b $\mathrm{b}^{+} \mathrm{Gr}-1^{\text {high }}$ neutrophils, CD $11 \mathrm{~b}^{+}$Siglec- $\mathrm{F}^{+}$eosinophils and $\mathrm{CD} 11 \mathrm{~b}^{+} \mathrm{F} 4 / 80^{+}$cells, but not in $\mathrm{CD}_{11} \mathrm{~b}^{+} \mathrm{CD} 11 \mathrm{c}^{+}$cells, within the colonic lamina propria of WT mice (figure 2A, B). DSS treatment upregulated LMIR3 in colonic lamina propria mast cells, while the same treatment did not influence LMIR3 expression in other myeloid cells tested (figure 2A, B). In light of the fact that the ATP-P2X7 signalling in mast cells contributes to colonic inflammation, ${ }^{8} 22$ we investigated $\mathrm{P} 2 \mathrm{X} 7$ expression in colonic lamina propria mast cells of WT or LMIR3 ${ }^{-/}$mice. Neither LMIR3 deficiency nor DSS treatment altered surface expression levels of P2X7 in colonic lamina propria mast cells (figure 2A). We then determined the percentage of $\mathrm{FcERI}^{+} \mathrm{c}-\mathrm{kit}^{+}$mast cells in $\mathrm{CD} 45^{+}$ LPMNCs of WT or $L M I R 3^{-/}$mice before and after DSS 
A

Control

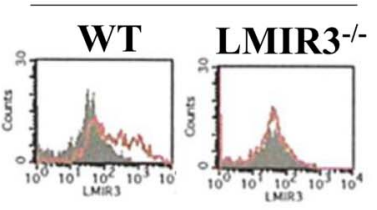

P2X7
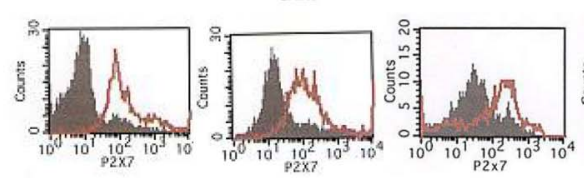

Control
DSS
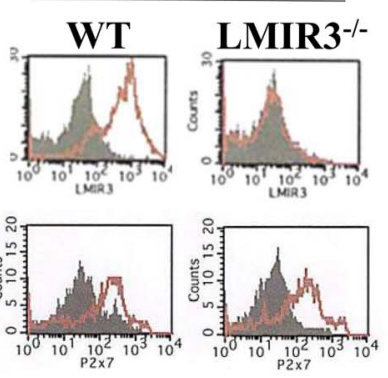

DSS

B
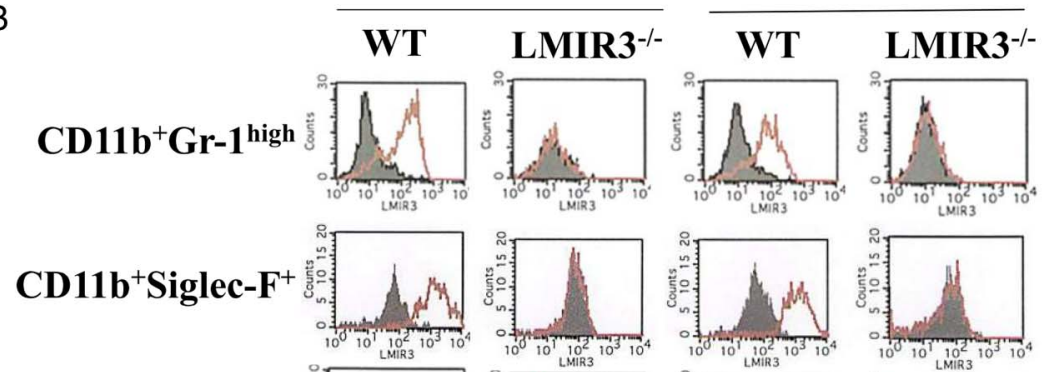

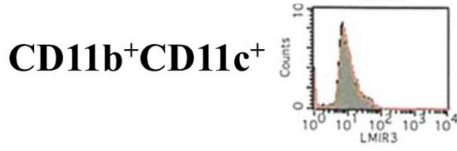
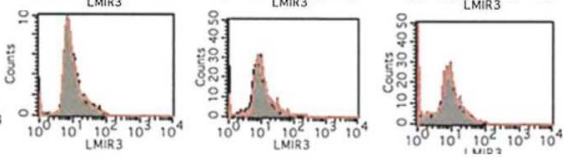

CD11b $^{+} \mathbf{F 4} / 80^{+}$
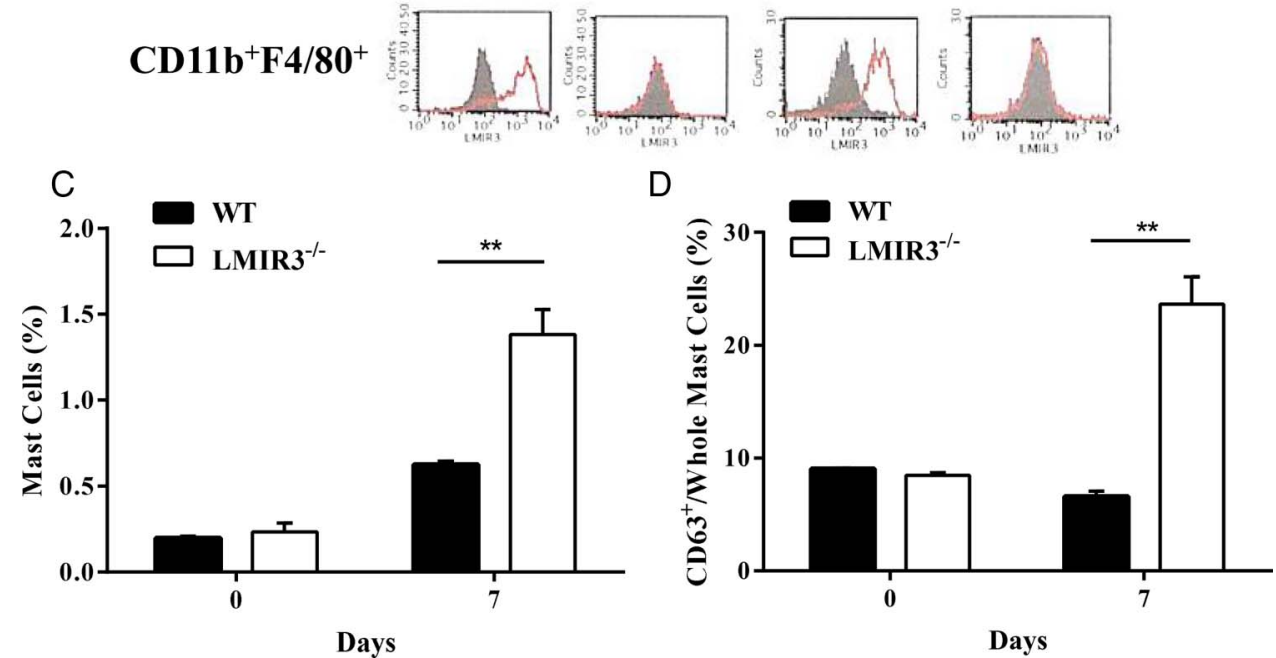

Figure 2 Dextran sodium sulfate (DSS) treatment induces a robust increase in degranulated mast cells in the colon of leucocyte mono-immunoglobulin-like receptor $3(L M I R 3)^{-/-}$mice. (A-D) Wild-type (WT) or LMIR3 ${ }^{-1-}$ mice on the C57BL/6 background (each, $\left.n=4\right)$ were subjected to the $1.5 \%$ DSS-induced colitis model. Flow cytometric analysis was performed on days 0 and 7 after DSS treatment. (A) Surface expression of LMIR3 or P2X7 in colonic lamina propria mast cells. (B) Surface expression of LMIR3 in CD11 ${ }^{+}$Gr- $1^{\text {high }}$ neutrophils, CD11 $b^{+}$Siglec- $F^{+}$ eosinophils, CD11 $\mathrm{b}^{+} \mathrm{CD} 11 \mathrm{c}^{+}$cells or $\mathrm{CD} 11 \mathrm{~b}^{+} \mathrm{F} 4 / 80^{+}$cells within the colonic lamina propria. (C) Percentages of mast cells in $\mathrm{CD}^{+} 5^{+}$lamina propria mononuclear cells. (D) Percentages of $\mathrm{CD} 3^{+}$colonic lamina propria mast cells. (C and D) Data are expressed as the mean $\pm \mathrm{SEM} .{ }^{* *} \mathrm{p}<0.01$ (Student's $t$ test). (A-D) The data are from one experiment which is representative of the other two experiments performed.

exposure. The percentages of colonic lamina propria mast cells were higher in DSS-treated $L M I R 3^{-/-}$mice than in WT counterparts, although those were increased in both types of mice after DSS exposure (figure 2C). In addition, DSS-treated LMIR3-/mice, but not WT mice, exhibited elevated percentages of $\mathrm{CD} 3^{+}$degranulated mast cells in the colonic lamina propria (figure 2D). ${ }^{8}$ Thus, LMIR3 deficiency caused the increase of activated mast cells in the colonic lamina propria of $1.5 \%$ DSS-treated mice, which was correlated with the severity of DSS-induced colitis.
DSS-treated $L M I R 3^{-/-}$mice exhibit enhanced colonic inflammation with a massive infiltration of inflammatory cells into the colon

We then examined the infiltration of inflammatory cells into the colon of DSS-treated mice. Consistent with histological observations, $1.5 \%$ DSS treatment increased the percentage of neutrophils in $\mathrm{CD}_{4} 5^{+}$LPMNCs, which were markedly higher in

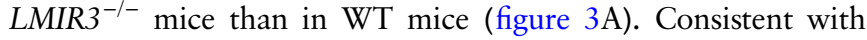
these results, we found a marked increase in myeloperoxidase activity related to neutrophil number in the colon of 
mono-immunoglobulin-like receptor 3

(LMIR3) deficiency enhances colonic

inflammation with a massive infiltration

of inflammatory cells in dextran sodium

sulfate (DSS)-treated mice. (A-F)

the C57BL/ 6 background (each, $n=4$ )

were subjected to the $1.5 \%$

DSS-induced colitis model. (A, C and D)

Percentages of (A) CD11 $b^{+}$Gr- $1^{\text {high }}$

neutrophils, (C) CD11 b ${ }^{+}$Siglec- $\mathrm{F}^{+}$

eosinophils or (D) CD11 b ${ }^{+}$CX3CR $1^{\text {int }}$

cells in $\mathrm{CD} 45^{+}$lamina propria

mononuclear cells (LPMNCS) or (B)

colonic tissue neutrophils estimated by

measuring myeloperoxidase activity

from the mice on days 0 and 7 .

( $E$ and F) Transcript levels of cytokines

and chemokines quantified by real-time

reverse transcription PCR analysis in

colonic LPMNCs (E) or protein levels of

cytokines and chemokines measured by

ELISA in colon explant culture

supernatants $(F)$ from the mice on days

0 and 7. (A-F) Data are expressed as

mean \pm SEM. ${ }^{*} p<0.05,{ }^{* *} p<0.01$ and

${ }^{* * *} p<0.001$ (Student's $t$ test). The data

are from one experiment which is

representative of the other three

experiments performed. IL, interleukin;

TNF, tumour necrosis factor.
Figure 3 Leucocyte

Wild-type (WT) or LMIR3 ${ }^{-1-}$ mice on

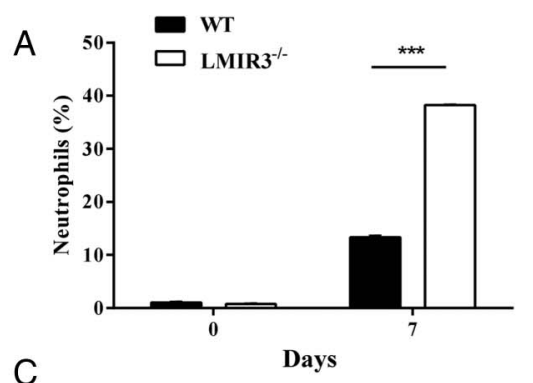

C
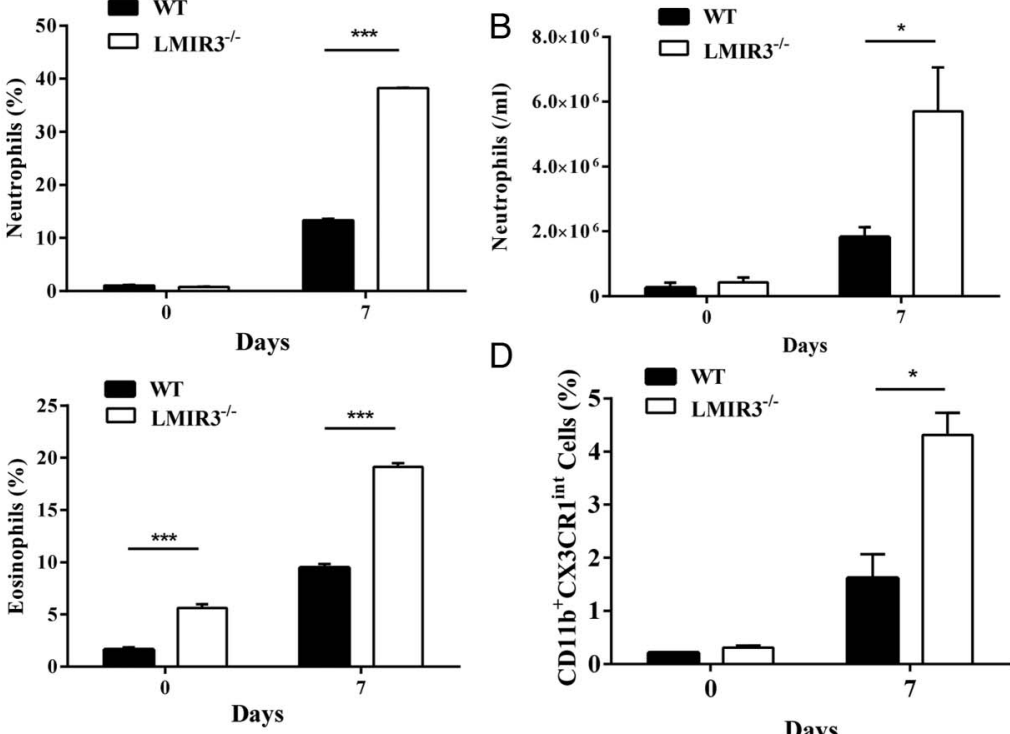

$\mathrm{D}$

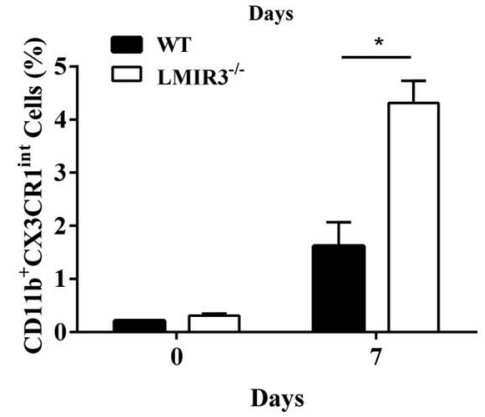

$E$
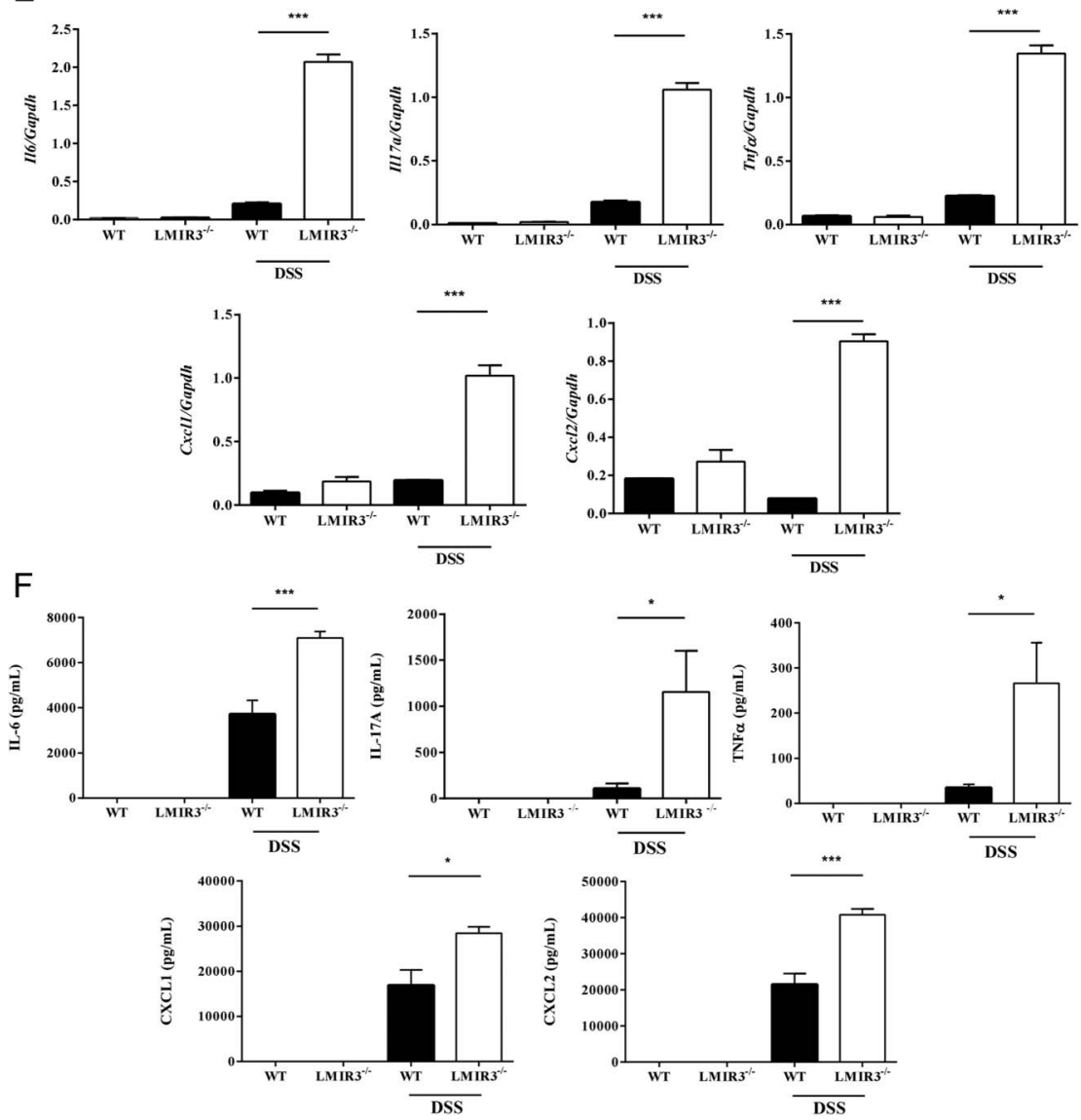

DSS-treated $\mathrm{LMIR}^{-/-}$mice (figure 3B). As reported, ${ }^{24}$ the percentages of eosinophils in CD45 ${ }^{+}$LPMNCs were higher in $L M I R 3^{-/-}$mice on the C57BL/6 background than in WT mice before DSS exposure (figure 3C). Although 1.5\% DSS treatment increased the percentage of colonic lamina propria eosinophils in both types of mice, DSS-treated $L M I R 3^{-1-}$ mice exhibited a remarkable increase in eosinophil populations (figure 3C). Notably, colonic lamina propria eosinophil numbers were not different between WT and $L M I R 3^{-/-}$mice on the BALB/c background under steady-state conditions, whereas DSS treatment strongly increased these eosinophil populations in $L M I R 3^{-/-}$ mice as compared with WT mice (see online supplementary figure S4). These results implied that the observed inflammatory differences between WT and $L M I R 3^{-/-}$mice were independent of the difference in eosinophils. Analysis of CD $11 b^{+} \mathrm{CX} 3 \mathrm{CR} 1^{\text {int }}$ cells in $\mathrm{CD}_{4} 5^{+}$LPMNCs showed that the percentages of these cell populations were also increased by DSS treatment in both types of mice, while those were higher in $L M I R 3^{-/-}$mice than in WT mice (figure 3D). ${ }^{11}$ Consistent with a robust infiltration of inflammatory cells in the colon of DSS-treated LMIR3 ${ }^{-/-}$ mice, real-time reverse transcription PCR analysis of mRNA levels displayed higher amounts of inflammatory cytokines (interleukin-6 (IL-6), IL-17A or tumour necrosis factor $\alpha$ ) and chemokines (CXCL1 or CXCL2) in colonic LPMNCs of 
DSS-treated $L M I R 3^{-/-}$mice compared with WT mice (figure 3E). Similarly, ex vivo colon punch biopsy cultures of DSS-treated $L M I R 3^{-/-}$mice exhibited remarkably increased levels of inflammatory cytokines and chemokines at the protein levels as compared with those of DSS-treated WT mice (figure 3F). Thus, $1.5 \%$ DSS treatment caused remarkably more severe colonic inflammation with an enhanced infiltration of inflammatory cells in $L M I R 3^{-/-}$mice.

$\mathrm{LMIR3}^{-/-}$mast cells contribute to an exacerbation of DSS-induced colitis

We then asked which cell populations were responsible for the high susceptibility of $L M I R 3^{-/-}$mice to DSS-induced colitis.
First, we used $1.5 \%$ DSS to induce colitis in lethally irradiated WT mice adoptively transferred with WT or $L M I R 3^{--}$bone marrow (BM). The results show that $L M I R 3^{-/-}$BM-transplanted mice suffered from more severe colitis with greater weight loss and colon shortening compared with WT BM-transplanted mice (figure 4A, B), implicating $L M I R 3^{-/-}$BM-derived cells in the exacerbation of colitis of $L M I R 3^{-/-}$mice. We next used the same model in mast cell-deficient Kit ${ }^{W-s h / W-s h}$ and Kit ${ }^{W-s h / W-s h} L M I R 3^{-/-}$ mice. Kit ${ }^{W-s h / W-s h}$ mice exhibited mild body weight loss and colon shortening at levels comparable with those seen in WT mice (figure 4C, D). LMIR3 deficiency did not influence the severity of DSS-induced colitis in $\mathrm{Kit}^{\mathrm{W}-\mathrm{sh} / \mathrm{W}-\mathrm{sh}}$ mice (figure 4C, D). We further compared the severity of $1.5 \%$ DSS-induced colitis
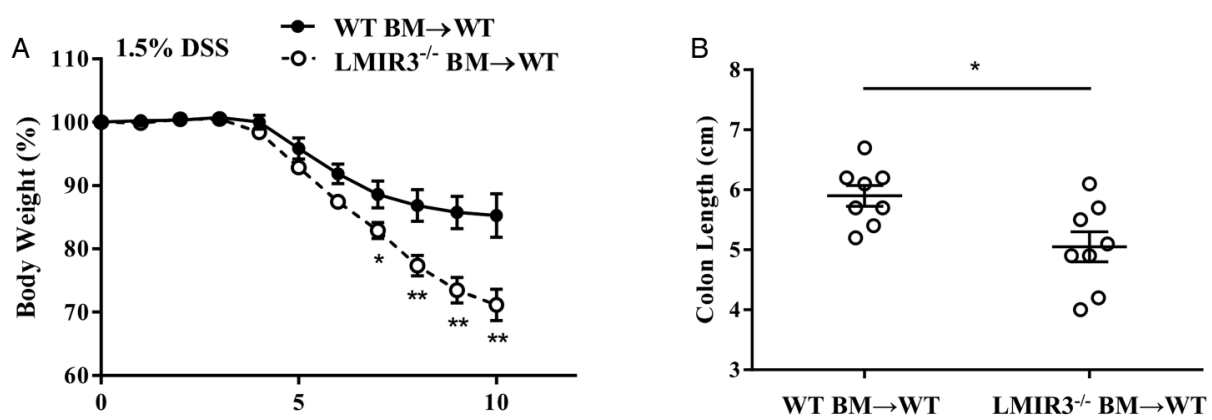

C
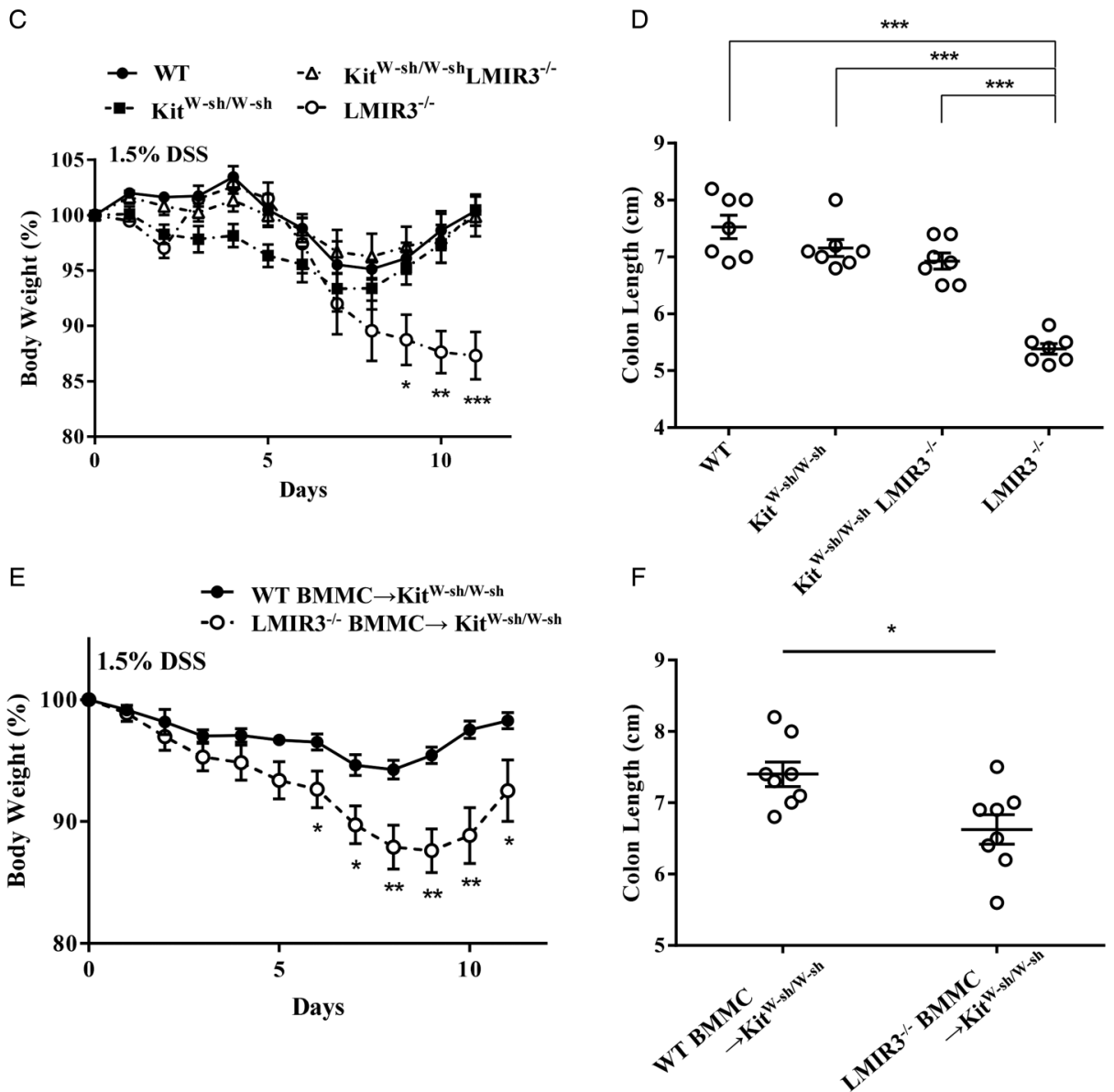

Figure 4 Leucocyte mono-immunoglobulin-like receptor $3(L M I R 3)^{-/-}$mast cells contribute to an exacerbation of dextran sodium sulfate (DSS)-induced colitis. (A-F) Mice on the C57BL/6 background were subjected to the $1.5 \%$ DSS-induced colitis model. (A and B) Wild-type (WT) mice

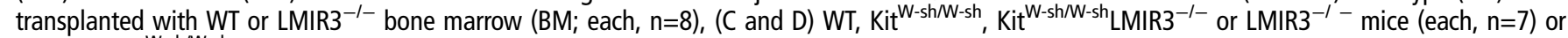
( $E$ and F) Kit ${ }^{W-s h / W-s h ~}$ transplanted with WT or LMIR3-deficient bone marrow-derived mast cells (BMMCs; each, $n=8$ ) were used. (A, C and F) Body weight. ( $B, D$ and F) Colon length on day 7 after DSS treatment. Data are expressed as the mean $\pm S E M .{ }^{*} p<0.05,{ }^{* *} p<0.01$ and ${ }^{* * *} p<0.001$ (Student's $t$ test). The data are from one experiment which is representative of the other one experiment performed. 
between $K i t^{W-s h / W-s h}$ mice adoptively transferred with WT or LMIR3 $^{-/-}$BMMCs. Colonic lamina propria mast cells were detected at comparable levels in these two types of BMMC-transplanted mice (see online supplementary figure S5). Transplantation with LMIR3-deficient mast cells, but not with WT mast cells, remarkably increased the severity of colitis (causing body weight loss and colon shortening) in $\mathrm{Kit}^{\mathrm{W}-\mathrm{sh} / \mathrm{W}-\mathrm{sh}}$ mice (figure $4 \mathrm{E}, \mathrm{F}$ ). Consistently, Kit ${ }^{W-s h / W-s h}$ with LMIR3deficient mast cells, but not with WT mast cells, exhibited a marked increase in neutrophils and eosinophils in $\mathrm{CD} 45^{+}$ LPMNCs (see online supplementary figure S6). In contrast, we found that WT mice were more susceptible to $2.5 \%$ DSS-induced colitis than $K i t^{W-s h / W-s h}$ mice (data not shown), which is in agreement with a previous report. ${ }^{8}$ In this model, Kit ${ }^{W-s h / W-s h} L M I R 3^{-/-}$mice developed only mild colitis with phenotypes similar to $K i t^{W-s h / W-s h}$ mice (see online supplementary figure S7). Transplantation of BMMCs, irrespective of LMIR3 expression, into $\mathrm{Kit}^{\mathrm{W}-\mathrm{s} / \mathrm{W}-\mathrm{s} h}$ mice increased the severity of $2.5 \%$ DSS-induced colitis; however, Kit ${ }^{W-s h / W-s h}$ mice with LMIR3deficient mast cells exhibited more severe colitis with a higher lethality than those with WT mast cells did (see online supplementary figure S8). Thus, LMIR3-deficient mast cells seemed to play an important role in the exacerbation of DSS-induced colitis in $L M I R 3^{-/-}$mice.

\section{Ceramide-LMIR3 interaction inhibits ATP-stimulated mast cell activation}

To clarify the role of the ATP-P2X7 signalling axis in the exacerbation of colitis in $L M I R 3^{-/-}$mice, we measured ATP levels in the colon of WT or LMIR3 ${ }^{-/-}$mice after 1.5\% DSS exposure. DSS treatment gradually increased the ATP content of the colon of mice on days 3-7; ATP levels were comparable between both groups of mice on day 3, but were higher in $L M I R 3^{-/}$mice than in WT mice on day 7 (figure 5A). When mice were intrarectally injected with non-hydrolyzable adenosine 5'-O-(3-thio) triphosphate (ATP $\gamma \mathrm{S})$, this treatment increased the percentage of $\mathrm{CD} 3^{+}$colonic lamina propria mast cells of $L M I R 3^{-/-}$mice, but not of WT mice, under our experimental conditions (figure 5B). We then asked whether the ceramide-LMIR3 interaction suppresses ATP-stimulated mast cell activation. WT or $L M I R 3^{-/-}$ BMMCs were stimulated with ATP on plates coated with ceramide, PC or vehicle. Surface expression levels of P2X7 as well as FceRI and c-kit were comparable between WT and LMIR3 ${ }^{-/-}$ BMMCs (see online supplementary figure S9). ATP stimulation induced the release of $\beta$-hexosaminidase, a marker of degranulation, and the production of neutrophil chemoattractants, CXCL2 and leucotriene B4 (LTB4), at equivalent levels in WT and $L M I R 3^{-/-}$BMMCs in the absence of ceramide (figure $5 \mathrm{C}$ ). In contrast, ceramide-LMIR3 binding specifically inhibited $\beta$-hexosaminidase release as well as CXCL2 and LTB4 production of WT BMMCs, but not of $L M I R 3^{-/-}$BMMCs, in response to ATP (figure 5C). To test whether LMIR3 inhibitory motifs are involved in LMIR3-mediated inhibition of ATP-stimulated mast cell activation, $\mathrm{LMIR}^{-/-}$BMMCs were transduced with LMIR3 WT, LMIR3 (Y241F-Y289F-Y325F) mutant or empty vector. In this mutant, three tyrosine residues in the two ITIMs and a single ITSM of LMIR3 were replaced with phenylalanine residues. These BMMC transfectants exhibited equivalent levels of FceRI, c-kit and P2X7 (data not shown). Surface expression levels of the transduced LMIR3 were comparable between BMMCs transduced with LMIR3 WT or its mutant (data not shown). Measurement of $\beta$-hexosaminidase release showed that ATP-stimulated mast cell degranulation was inhibited only in LMIR3 WT-transduced BMMCs, but not in LMIR3
(Y241F-Y289F-Y325F) mutant-transduced BMMC, on ceramide-coated plates (figure 5D). Similarly, the production of proinflammatory cytokine IL-6 in WT BMMCs was suppressed only on plates coated with ceramide, but not with phosphatidylserine (PS), PC or vehicle (figure 5E). However, such inhibition in the presence of ceramide was not observed in $\mathrm{LMIR}^{-/}$ BMMCs stimulated by ATP (figure 5E). Confocal microscopic analysis showed that extracellular ceramide was distributed in the surroundings of mast cells in colonic tissues and tended to be increased by DSS treatment (figure 5F). Thus, DSS treatment strongly enhanced ATP-mediated activation of colonic mast cells in $L M I R 3^{-/-}$mice presumably due to the lack of inhibitory signals via the ceramide-LMIR3 interaction.

\section{DSS-induced colitis is aggravated by disrupting ceramide- LMIR3 binding, whereas it is suppressed by administration of ceramide liposomes}

To validate the protective role of the ceramide-LMIR3 interaction in DSS-induced colitis, WT or $L M I R 3^{-/-}$mice were treated with an anticeramide $\mathrm{Ab}$ or with a control $\mathrm{Ab}$ during $1.5 \%$ DSS exposure. Notably, treatment with anticeramide $\mathrm{Ab}$, but not with a control $\mathrm{Ab}$, increased the severity of colitis in WT mice at levels comparable with that found in $L M I R 3^{-/}$ mice (figure 6A-C).Conversely, treatment with the anticeramide $\mathrm{Ab}$ did not influence DSS-induced colitis in $\mathrm{LMIR}^{-/}$mice (figure 6A-C). Similarly, WT or LMIR3 ${ }^{-/-}$mice were administered with LMIR3-Fc or with a control Fc during $1.5 \%$ DSS exposure. LMIR3-Fc-treated WT mice developed more severe colitis with greater weight loss than control Fc-treated WT mice (figure 6D). We confirmed that LMIR3-Fc treatment failed to exacerbate DSS-induced colitis in $L M I R 3^{-/-}$mice (figure 6D). Thus, disrupting the ceramide-LMIR3 interaction aggravated DSS-induced colitis in WT mice. We finally tested whether treatment with ceramide liposomes improves DSS-induced colitis. To this end, we used $2.5 \%$ DSS to induce severe colitis in mice. WT mice treated with ceramide liposomes compared with control liposome exhibited an attenuated DSS-induced colitis with less weight loss (figure 6E). Neither treatment reversed the lethality of DSS-induced colitis in $L M I R 3^{-/-}$mice (figure $6 \mathrm{E}$ ). Consistent with these results, treatment with ceramide liposomes substantially inhibited colonic mast cell degranulation in WT mice, but not in $L M I R 3^{-/-}$mice, after DSS exposure (figure 6F). These results indicate that the ceramide liposome treatment inhibited ATP-mediated mast cell activation, thereby improving DSS-induced colitis in WT mice.

\section{DISCUSSION}

The development of colitis is temporally and spatially regulated by a variety of immune cells in concert with colonic epithelial cells. $^{2-4}$ 8-12 However, the key cellular signals that trigger exacerbation of colitis remained unclear until the recent finding that the ATP-P2X7 signalling axis in mast cells is pivotal to intestinal inflammation in experimental colitis. ${ }^{8}$ Based on this knowledge, we demonstrate in the present study that the ceramide-LMIR3 interaction suppresses experimental colitis by inhibiting ATP-mediated mast cell activation.

Comparative analysis of DSS-induced colitis between WT and $L M I R 3^{-/-}$mice demonstrated that $L M I R 3^{-/-}$mice were more prone to colonic inflammation than WT mice under the same experimental conditions including genetic background and the dose of DSS administration: low-dose (1.5\%) DSS treatment caused only mild colitis in WT C57BL/6 mice, but induced severe colitis in $\mathrm{LMIR3}^{-/-}$mice; high-dose (2.5\%) DSS treatment induced lethal colitis only in most $L M I R 3^{-/-} \mathrm{C} 57 \mathrm{BL} / 6$ mice. 

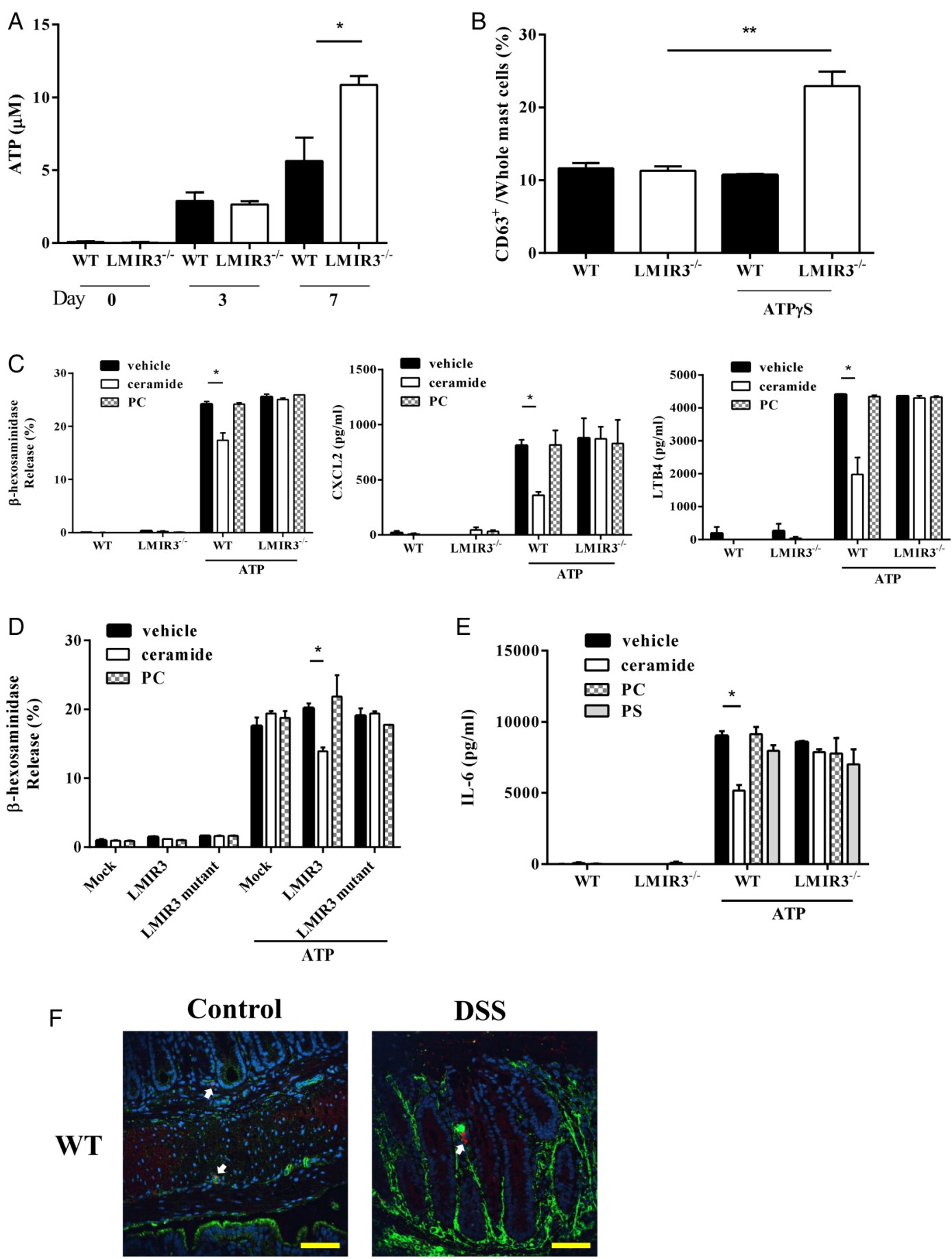

Figure 5 Ceramide-leucocyte mono-immunoglobulin-like receptor 3 (LMIR3) interaction inhibits ATP-stimulated mast cell activation. (A) Wild-type (WT) or LMIR3 ${ }^{-1-}$ mice on the C57BL/6 background (each, $n=3$ ) were subjected to $1.5 \%$ dextran sodium sulfate (DSS)-induced colitis model. The concentrations of ATP released by colonic tissues from the mice on days 0,3 or 7 . (B) WT or LMIR3 ${ }^{-1-}$ mice on the C57BL/6 background (each, $\mathrm{n}=3$ ) were intrarectally administered with ATP $\gamma$ S. Percentages of $\mathrm{CD} 3^{+}$colonic lamina propria from the mice on days 0 and 3 after administration. (C) WT or LMIR3 ${ }^{-l-}$ bone marrow-derived mast cells (BMMCs) were stimulated with $3 \mathrm{mM}$ ATP on plates coated with ceramide, phosphatidylcholine (PC) or vehicle. Release of $\beta$-hexosaminidase (left) or production of CXCL2 (middle) or LTB4 (right). (D) LMIR3 ${ }^{-l-}$ BMMCs transduced with LMIR3 WT, LMIR3 (Y241F-Y289F-Y325F) mutant, or empty vector (mock) were stimulated with $3 \mathrm{mM}$ ATP on plates coated with ceramide, PC or vehicle. Release of $\beta$-hexosaminidase. (E) WT or LMIR3 ${ }^{-1-}$ BMMCs were stimulated with $3 \mathrm{mM}$ ATP on plates coated with ceramide, PC, phosphatidylserine (PS) or vehicle. Production of interleukin (IL)-6. (A-E) Data are expressed as mean $\pm S E M$. ${ }^{*} p<0.05,{ }^{* *} p<0.01$ and ${ }^{* * *} p<0.001$ (Student's $t$ test). The data are from one experiment which is representative of the other two experiments performed. (F) Frozen colon sections of WT mice on days 0 and 7 after 1.5\% DSS treatment were stained with an anticeramide Ab (ceramides; green) and an anti-mast cell tryptase Ab (mast cell; red). The nuclei were counterstained with 4',6-diamidino-2-phenylindole (blue). White arrow indicates mast cell. The data are from one experiment which is representative of the other four experiments performed. Scale bars represent $100 \mu \mathrm{m}$. 

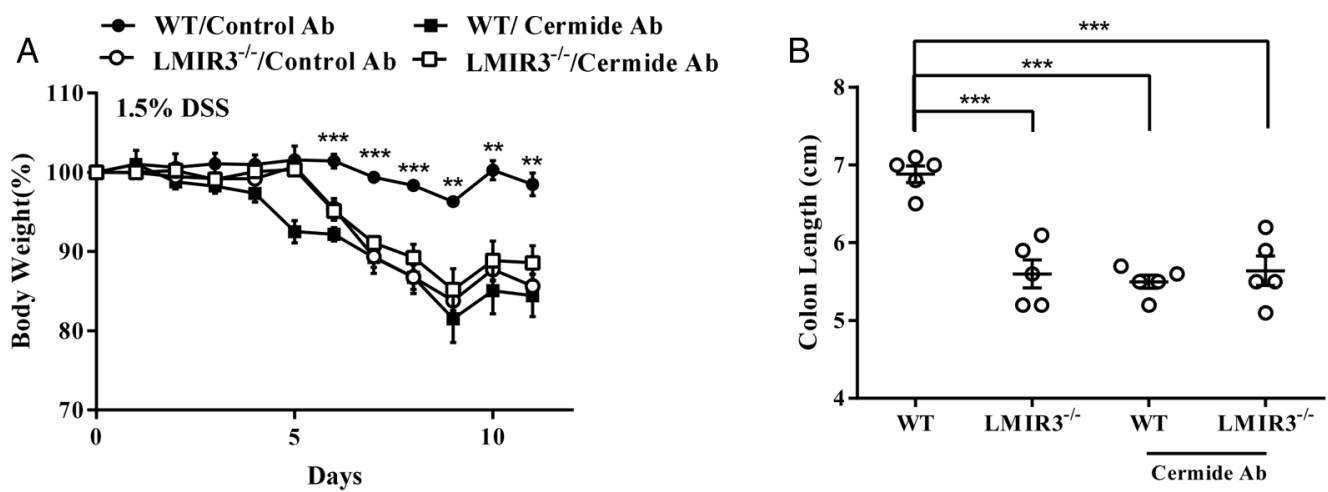

C

WT

Control

Ab

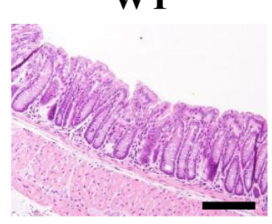

Ceramide

Ab

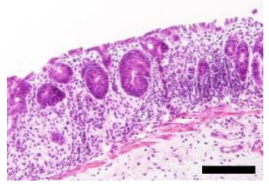

LMIR3 $^{-/-}$

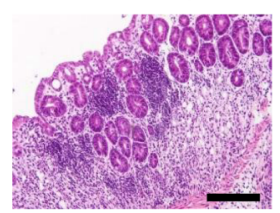

E

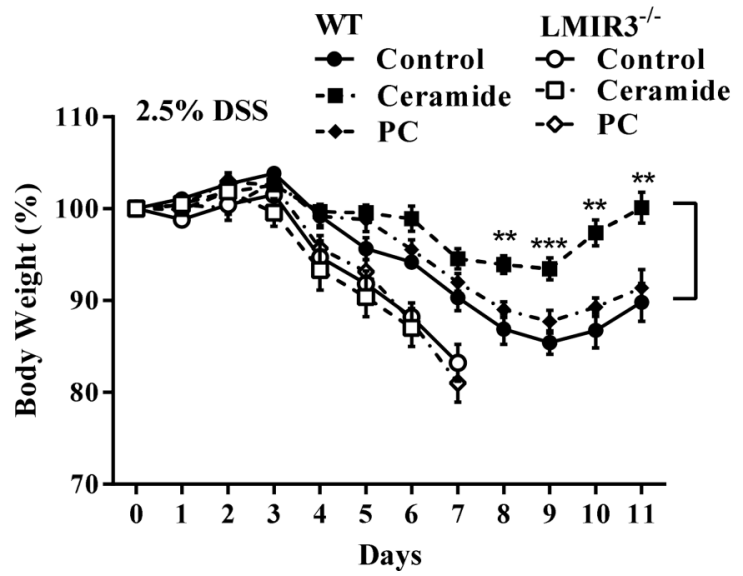

D

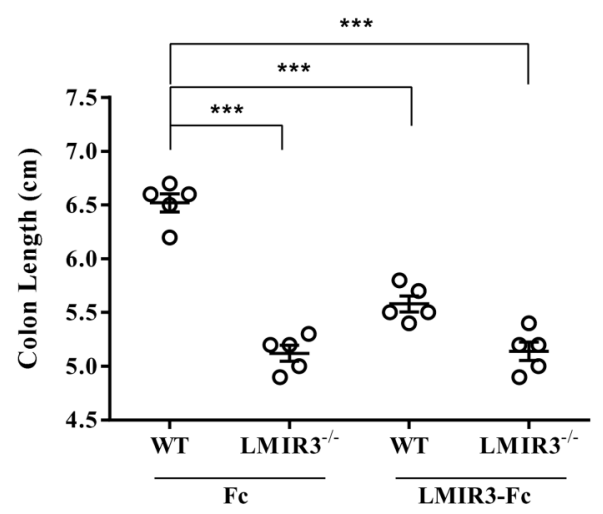

$\mathrm{F}$
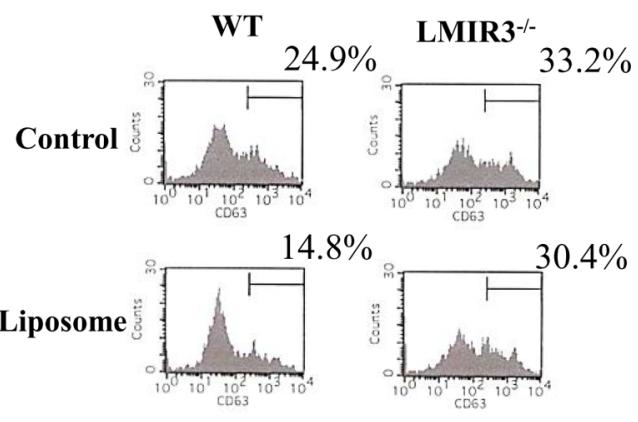

Figure 6 Dextran sodium sulfate (DSS)-induced colitis is aggravated by disrupting ceramide-leucocyte mono-immunoglobulin-like receptor 3 (LMIR3) binding, while that is suppressed by administration of ceramide liposomes. (A-C) Anticeramide Ab or control Ab-treated wild-type (WT) or $\mathrm{LMIR3}^{-1-}$ mice on the C57BL/6 background (each, $\mathrm{n}=5$ ) were subjected to the $1.5 \%$ DSS-induced colitis model. (A) Body weight. (B) Colon length on day 7. (C) Representative H\&E-stained colonic sections were shown. Scale bars represent $100 \mu \mathrm{m}$. (D) LMIR3-Fc-treated or control Fc-treated WT or $L M I R 3^{-/-}$mice on the C57BL/6 background ( $n=5$ per genotype) were subjected to the $1.5 \%$ DSS-induced colitis. Colon length on day 7 . ( $E$ and $F$ ) Ceramide or phosphatidylcholine (PC) liposomes or phosphate buffer saline-treated WT or LMIR3 ${ }^{-1}$ mice on a C57BL/6 background ( $n=10$ per genotype) were subjected to the $2.5 \%$ DSS-induced colitis. (E) Body weight. (F) Percentages of CD63 ${ }^{+}$colonic lamina propria mast cells on day 7. $\left(A, B, D\right.$ and $E$ ) Data are expressed as the mean \pm SEM. ${ }^{* *} p<0.01,{ }^{* * *} p<0.001$ (Student's $t$ test). (A-F) The data are from one experiment which is representative of the other one experiment performed.

Moreover, the high susceptibility of $L M I R 3^{-/-}$mice to TNBS-induced colitis underscored the importance of LMIR3 as a negative regulator of experimental colitis. Interestingly, treatment with low-dose DSS mildly increased neutrophils, eosinophils and $\mathrm{CD} 11 \mathrm{~b}^{+} \mathrm{CX} 3 \mathrm{CR} 1^{\text {int }}$ cells, which were involved in the development of colitis, ${ }^{8-11}$ in the colonic lamina propria of WT mice; however, the same treatment failed to significantly induce activation of colonic lamina propria mast cells expressing
LMIR3 as well as P2X7. Consistent with these results, the severity of low-dose DSS-induced colitis was influenced neither by mast cell deficiency in mice nor by reconstitution with WT mast cells in mast cell-deficient Kit $t^{W-s h / W-s h}$ mice. Thus, low-dose DSS treatment induced a mild infiltration of inflammatory cells into the colonic lamina propria, independently of mast cell activation. However, we found a prominent increase in activated mast cells in the colonic lamina propria of $L M I R 3^{-/-}$mice after 
low-dose DSS treatment. Importantly, similarly induced colitis in $K i t^{W-s h / W-s h}$ mice was profoundly enhanced by reconstitution with $L M I R 3^{-/-}$BMMCs, but not by crossing with $L M I R 3^{-/-}$ mice. Since LMIR3 deficiency did not affect P2X7 expression both in colonic lamina propria mast cells and in BMMCs, it is therefore plausible that LMIR3 deficiency in mast cells exacerbates colitis induced by low-dose DSS. In view of previous results, ${ }^{8}$ it is also possible to speculate that LMIR3 dampens colonic mast cell activation in response to relatively low levels of ATP produced in the colon injured by low-dose DSS. These assumptions may be supported by two of our observations: low-dose DSS treatment gradually elevated colonic ATP levels in proportion to the severity of colonic inflammation in mice; an intrarectal administration of ATP $\gamma$ S activated colonic lamina propria mast cells only in $L M I R 3^{-/}$mice. Meanwhile, in agreement with a previous report, ${ }^{8}$ we confirmed that high-dose DSS-treated $K i t^{W-s h / W-s h}$ mice compared with WT mice developed remarkably more mild colitis, which was aggravated by reconstitution with WT mast cells. At the same time, we found that highdose DSS-induced colitis in Kit ${ }^{W-s h / W-s h}$ mice was more profoundly aggravated by reconstitution with $L M I R 3^{-/-}$mast cells compared with WT mast cells, while it was not significantly influenced by LMIR3 deficiency. Collectively, it is possible to conclude that colonic mast cells contribute to the development of high-dose DSS-induced colitis in WT mice; severe injuries to the colon was followed by the release of high levels of ATP in the colon where ATP-mediated mast cell activation cannot be completely blocked by LMIR3. However, LMIR3-deficient colonic mast cells are pivotal in the exacerbation of colitis induced by DSS, irrespective of its dosage, presumably because those cells are fully activated due to the lack of LMIR3-mediated inhibitory signals. Consequently, LMIR3-deficient colonic mast cells released profoundly high levels of soluble factors that recruit inflammatory cells as well as of inflammatory mediators that directly promote tissue damage, all of which amplify intestinal inflammation. To clarify the possible role of LMIR3 deficiency in myeloid cells except mast cells in experimental colitis, further investigation will be required; a recent study reported that eosinophil-mediated signalling attenuates experimental colitis. ${ }^{25}$

Analysis of WT or LMIR3 ${ }^{-/-}$BMMCs demonstrated that, like high-affinity IgE receptor signalling, P2X7 signalling via exogenous ATP was inhibited by the binding of ceramide to LMIR3 in BMMCs. ${ }^{15}$ Note that other lipids tested, including PS which was reported to bind to CD300f/LMIR3, did not act as a functional ligand for CD300f/LMIR3 in this setting. ${ }^{26}$ In addition, the LMIR3(Y241F-Y289F-Y325F) mutant failed to inhibit ATP-stimulated activation of the transduced BMMCs in the presence of ceramide. These results therefore suggest that ceramide-LMIR3 binding inhibited ATP-mediated mast cell activation through tyrosine-phosphorylation of the two ITIMs and a single ITSM, which was able to induce recruitment of tyrosine phosphatases. ${ }^{14}$ As revealed by histochemical examination, extracellular ceramide was distributed in the surroundings of colonic mast cells. Moreover, administration of an anticeramide $\mathrm{Ab}$ or LMIR3-Fc, both of which disrupt ceramide-LMIR3 binding in colonic mast cells, aggravated DSS-induced colitis in WT mice, but not in $L M I R 3^{-/-}$mice. These in vitro and in vivo data highlight the importance of ceramide-LMIR3 binding in colonic mast cells in suppressing experimental colitis. Intriguingly, DSS treatment upregulated LMIR3 in colonic mast cells, while the same treatment tended to increase extracellular ceramide in colonic tissues possibly in association with tissue damage. These two events may co-operate to prevent excessive activation of colonic mast cells in this model. Most importantly, intravenous administration of ceramide liposomes, which was thought to possibly increase extracellular ceramide in colonic tissues, attenuated DSS-induced colitis, although more effective and safe treatment against colitis with ceramide liposomes, including the dose, route and frequency, remains to be determined. Given that intestinal mast cell activation via extracellular ATP is implicated in the pathogenesis of certain types of human IBD, ${ }^{8}$ LMIR3-targeted ceramide liposomes would provide novel therapeutic strategies for IBD.

In conclusion, LMIR3 inhibits ATP-mediated colonic mast cell activation through its interaction with ceramide, thereby attenuating experimental colitis. Accordingly, treatment with ceramide liposomes ameliorates colonic inflammation in mouse models of IBD.

Acknowledgements We thank Yosuke Kurashima (The University of Tokyo) for helpful discussions.

Contributors TM performed all the experiments and participated in writing the manuscript. $\mathrm{KI}, \mathrm{MI}, \mathrm{MT}, \mathrm{AM}, \mathrm{YY}$ and $\mathrm{AK}$ assisted with the experiments. $\mathrm{KO}$ and TT analysed the data. TK and JK conceived the project, analysed the data and actively participated in manuscript writing.

Funding This study was supported by JSPS KAKENHI Grant Number 23390257 and 26293231 and in part by Grant-in-Aid for JSPS Fellows (26000014).

\section{Competing interests None.}

Provenance and peer review Not commissioned; externally peer reviewed.

Open Access This is an Open Access article distributed in accordance with the Creative Commons Attribution Non Commercial (CC BY-NC 4.0) license, which permits others to distribute, remix, adapt, build upon this work non-commercially, and license their derivative works on different terms, provided the original work is properly cited and the use is non-commercial. See: http://creativecommons.org/ licenses/by-nc/4.0/

\section{REFERENCES}

1 Cader MZ, Kaser A. Recent advances in inflammatory bowel disease: mucosal immune cells in intestinal inflammation. Gut 2013:62:1653-64.

2 Kaser A, Zeissig S, Blumberg RS. Inflammatory bowel disease. Annu Rev Immunol 2010;28:573-621.

3 Saleh M, Trinchieri G. Innate immune mechanisms of colitis and colitis-associated colorectal cancer. Nat Rev Immunol 2011;11:9-20.

4 Fukata $M$, Arditi $M$. The role of pattern recognition receptors in intestinal inflammation. Mucosal Immunol 2013;6:451-63.

5 Wirtz S, Neufert C, Weigmann B, et al. Chemically induced mouse models of intestinal inflammation. Nat Protoc 2007;2:541-6.

6 Cooper HS, Murthy SN, Shah RS, et al. Clinicopathologic study of dextran sulfate sodium experimental murine colitis. Lab Invest 1993;69:238-49.

7 Rijnierse A, Koster AS, Nijkamp FP, et al. Critical role for mast cells in the pathogenesis of 2,4-dinitrobenzene-induced murine colonic hypersensitivity reaction. J Immunol 2006;176:4375-84.

8 Kurashima Y, Amiya T, Nochi T, et al. Extracellular ATP mediates mast cell-dependent intestinal inflammation through P2X7 purinoceptors. Nat Commun 2012;3:1034.

9 Hamilton MJ, Sinnamon MJ, Lyng GD, et al. Essential role for mast cell tryptase in acute experimental colitis. Proc Natl Acad Sci USA 2011;108:290-5.

10 Waddell A, Ahrens R, Steinbrecher K, et al. Colonic eosinophilic inflammation in experimental colitis is mediated by Ly6C ${ }^{\text {high }} \mathrm{CCR}^{+}$inflammatory monocyte/ macrophage-derived CCL11. J Immunol 2011;186:5993-6003.

11 Zigmond E, Varol C, Farache J, et al. Ly6C hi monocytes in the inflamed colon give rise to proinflammatory effector cells and migratory antigen-presenting cells. Immunity 2012;37:1076-90.

12 Rakoff-Nahoum S, Paglino J, Eslami-Varzaneh F, et al. Recognition of commensal microflora by toll-like receptors is required for intestinal homeostasis. Cell 2004:118:229-41.

13 Bischoff SC. Role of mast cells in allergic and non-allergic immune responses: comparison of human and murine data. Nat Rev Immunol 2007;7:93-104.

14 Izawa K, Yamanishi Y, Maehara A, et al. The receptor LMIR3 negatively regulates mast cell activation and allergic responses by binding to extracellular ceramide. Immunity 2012;37:827-39.

15 Nakahashi-Oda C, Tahara-Hanaoka S, Shoji M, et al. Apoptotic cells suppress mast cell inflammatory responses via the CD300a immunoreceptor. J Exp Med 2012;209:1493-503.

16 Colonna M. TREMs in the immune system and beyond. Nat Rev Immunol 2003;3:445-53. 
17 Chung DH, Humphrey MB, Nakamura MC, et al. CMRF-35-like molecule-1, a novel mouse myeloid receptor, can inhibit osteoclast formation. J Immunol 2003;171: 6541-8.

18 Izawa K, Kitaura J, Yamanishi Y, et al. Functional analysis of activating receptor LMIR4 as a counterpart of inhibitory receptor LMIR3. J Biol Chem 2007;282:17997-8008.

19 Grimbaldeston MA, Chen CC, Piliponsky AM, et al. Mast cell-deficient W-sash c-kit mutant Kit W-sh/W-sh mice as a model for investigating mast cell biology in vivo. Am J Pathol 2005;167:835-48.

20 Kitaura J, Song J, Tsai M, et al. Evidence that lgE molecules mediate a spectrum of effects on mast cell survival and activation via aggregation of the FcepsilonRI. Proc Natl Acad Sci USA 2003;100:12911-16.

21 Weigmann B, Tubbe I, Seidel $D$, et al. Isolation and subsequent analysis of murine lamina propria mononuclear cells from colonic tissue. Nat Protoc 2007;2:2307-11.
22 Surprenant A, North RA. Signaling at purinergic P2X receptors. Annu Rev Physiol 2009;71:333-59.

23 Furuno T, Teshima R, Kitani S, et al. Surface expression of CD63 antigen (AD1 antigen) in P815 mastocytoma cells by transfected IgE receptors. Biochem Biophys Res Commun 1996;219:740-4.

24 Moshkovits I, Shik D, Itan M, et al. CMRF35-like molecule 1 (CLM-1) regulates eosinophil homeostasis by suppressing cellular chemotaxis. Mucosal Immunol 2014;7:292-303.

25 Masterson JC, McNamee EN, Fillon SA, et al. Eosinophil-mediated signaling attenuates inflammatory responses in experimental colitis. Gut 2015;64: 1236-47.

26 Choi SC, Simhadri VR, Tian L, et al. Cutting edge: mouse CD300f (CMRF-35-like molecule-1) recognizes outer membrane-exposed phosphatidylserine and can promote phagocytosis. J Immunol 2011;187:3483-7. 